\title{
Facilitating the creation of interactive multi-device Learning Objects using an online authoring tool
}

\author{
Aldo Gordillo, Enrique Barra and Juan Quemada \\ Escuela Técnica Superior de Ingenieros de Telecomunicación \\ Universidad Politécnica de Madrid \\ Avenida Complutense 30, 28040, Madrid, Spain \\ \{agordillo, ebarra,jquemada\}@dit.upm.es
}

\begin{abstract}
Learning Objects facilitate reuse leading to cost and time savings as well as to the enhancement of the quality of educational resources. However, teachers find it difficult to create or to find high quality Learning Objects, and the ones they find need to be customized. Teachers can overcome this problem using suitable authoring systems that enable them to create high quality Learning Objects with little effort. This paper presents an open source online e-Learning authoring tool called ViSH Editor together with four novel interactive Learning Objects that can be created with it: Flashcards, Virtual Tours, Enriched Videos and Interactive Presentations. All these Learning Objects are created as web applications, which can be accessed via mobile devices. Besides, they can be exported to SCORM including their metadata in IEEE LOM format. All of them are described in the paper including an example of each. This approach for creating Learning Objects was validated through two evaluations: a survey among authors and a formal quality evaluation of 209 Learning Objects created with the tool. The results show that ViSH Editor facilitates educators the creation of high quality Learning Objects.
\end{abstract}

Keywords - authoring tool; learning objects; evaluation

\section{INTRODUCTION}

Educational technologies provide educators with alternative ways of presenting material, engaging students and improving academic performance. The adoption and evolution of this type of technologies have boosted the use of technology enhanced learning (TEL) in many levels of education. Learning Objects (LOs) are acquiring increasing relevance since they are becoming the building blocks of TEL. LOs facilitate reuse and interoperability [1] leading to minimization of production cost, time saving and quality enhancement of digital learning experiences [2]. The multiple benefits of using LOs have been exposed by several empirical researches ([3], [4], [5], [6], [7]) that have examined their instructional effectiveness and learning outcomes across different educational environments.

There are still two main barriers preventing more extensive and effective use of these objects. First, teachers find it difficult to create high quality LOs [8], and second, they also have difficulties to locate high quality LOs, and the ones they end up finding usually need to be customized to be adapted to their needs [9]. A teacher can search on an educational repository and find an outstanding LO, but if it is too difficult or easy for their students or if it is in a language they cannot understand, its effectiveness to support learning might be drastically reduced. To mitigate the searching problem, Learning Object Repositories (LORs) use ranking metrics and sorting algorithms to order the search results ([10], [11], [12]). Another approach to help educators to find suitable resources is the use of LO recommender systems ([13], [14]). However, these solutions by themselves are insufficient to generalize the use of LOs since educators are limited to the resources stored in the repositories and their content cannot be customized.

A more powerful solution to effectively help teachers to overcome these barriers is the use of e-Learning authoring tools. These applications allow to create high quality LOs with little effort and low cost, without requiring authors any knowledge about programming. Besides the creation of custom content, authoring tools also allow to repurpose and adapt existing contents. However, not all authoring tools are equally effective. The success or failure of this approach will depend on two key factors. Firstly, on the extent to which the intended authors find the authoring tool easy to use, and secondly, on the quality as educational resources of the created LOs.

This paper presents an open source online e-Learning authoring tool called ViSH Editor, which allows to create four novel interactive web LOs: Flashcards, Virtual Tours, Enriched Videos and Interactive Presentations. These LOs are also explained including an example of each of them. Both the authoring tool and the created LOs are available online through the Virtual Science Hub (http://vishub.org) [15], an open source e-Learning platform developed as part of the GLOBAL excursion European project. Since the platform's launch one year and a half ago, many teachers have used the tool and hundreds of LOs have been created and used in different educational settings.

This approach for creating LOs has been validated through two evaluations. Firstly, a survey was conducted to collect authors' feedback. Secondly, a total of 209 LOs created with ViSH Editor were evaluated using LORI [16], a formal LO evaluation model. The results of the evaluations show that ViSH Editor facilitates the creation of high quality LOs.

The rest of the paper is organized as follows. The next section reviews related work of authoring tools as well as LOs. Section 3 provides an overview of the ViSH Editor authoring tool. Section 4 explains each of the four aforementioned LOs. Section 5 shows the results of the evaluations carried out. Finally, section 6 finishes with some conclusions together with an outlook on future work. 


\section{RELATED WORK}

An e-Learning authoring tool can be defined as an application that enables authors to create complete educational resources (or courseware) by integrating and linking together different objects (such as a paragraph of text or a picture) and custom content generated by them. Authors can define the relationships among the objects and customize their sequencing. Most authoring systems also make possible to repurpose and adapt existing resources. These systems are usually designed to be simple, easy to use and suitable for users with low computer skills. Thereby, e-Learning authoring tools allow educators without programming knowledge to engage in educational software development. Nevertheless, some of these tools also support programming languages to generate more sophisticated applications. Other similar definitions of the term can be found in [17], [18], [19] and [20].

The key benefits of authoring tools for creating educational resources are cost and time saving. Most educational institutions cannot afford the development of custom applications, and therefore these tools play a vital role in the creation and spreading of educational software. It is worth pointing out that authoring systems can facilitate authors to create high quality educational resources, but they do not guarantee it [18]. Even the perfect authoring tool would produce poor courseware if the author lacks the necessary knowledge or does not create the courseware following basic educational principles. Nevertheless, we can affirm that authoring systems are able to significantly improve the levels of accessibility, reusability and interoperability, since the produced resources usually can be exported or are built conforming to some e-Learning standard such as SCORM [21] or TIN CAN API [22] and also they can be delivered ready to be visualized through multiple devices such as mobile phones or tablets. Summarizing, the aim any authoring tool should try to achieve is to be easy of use and facilitate the creation of high quality products [23].

Several studies have addressed the question of what criteria should be used to classify authoring tools [23] and to choose one based on the author's particular needs ([24], [25], [26]). Despite there is a lack of a universally recognized authoring tools classification system, some criteria can be used to characterize them. The most important one is the type of educational resource that can be created with the tool. Authoring tools can be used to create quizzes (e.g. Articulate Quizmaker [27]), presentations (e.g. Microsoft PowerPoint [28]), intelligent tutoring systems (e.g. REDEEM [29]), educational games (e.g. SGAME [30]) or even full e-Learning courses (e.g. Easy generator [31]). Other aspects that should be taken into account to choose an authoring tool are among others: platform support, media and files type support, automated programming, ease of use and creative freedom, support for assessment questions, interoperability of the created resources, extensibility and its cost and license. Another issue that has been tackled is how to compare different authoring tools. To do that, Preclík [18] established three criteria: power (what can be realized in the system), productivity (how much of the output can create the author per one unit of time) and ease of use (how long the author must learn how to work with the system).
Modern e-Learning authoring tools are increasingly relying more on the LO approach, focusing on increase the reuse capability of their resources. Examples of this are, among others, Reload [32], LOC [33], GLO Maker [34], LO Creator [35] and Educaplay [36]. Significant discussion has been devoted to establishing a universally accepted definition of LOs across the last years [37], however, consensus has not been reached yet. LOs are defined in this paper as "reusable digital resources tagged with metadata that are self-contained and that can be used for education". This definition is based on previous approaches to define LOs ([37], [38], [39], [40]). The potential of a LO for reuse increases as its size or granularity decreases [41], thereby they should be sufficiently large to be of educational value but also be small enough to be effectively reused [42]. LOs can be combined among them to build more complex ones forming a hierarchy. These LOs are known as granular LOs and the different hierarchy levels are called aggregation or granularity levels. LO models define these levels of granularity and specify how the components can be aggregated as well as the properties of these components [43]. LOs are tagged with metadata, where a description of the object is provided to improve their reusability, interoperability and discoverability. The most used LO metadata standards are Dublin Core [44] and IEEE LOM [45]. The LOM (Learning Object Metadata) standard allows to define application profiles (e.g. CanCore [46]) to refine the original specification to make it more suitable for its application by a particular community.

\section{AUTHORING TOOL}

ViSH Editor is a web-based e-Learning authoring tool that aims to facilitate the creation of high quality multi-device LOs. It is open source and the code is available at http://github.com/ging/vish_editor.

Fig. 1 shows the LO model followed by ViSH Editor, which defines four levels of granularity. This structure is quite similar to the one defined by the LOM standard [45] but with a few differences.

- The first level, which corresponds to the most granular or atomic level, includes raw media files like images, audios, videos or flash objects and single elements such as paragraphs of text, documents or websites. More sophisticated elements such as quizzes or web applications (e.g. SCORM packages) belong to the first level too.

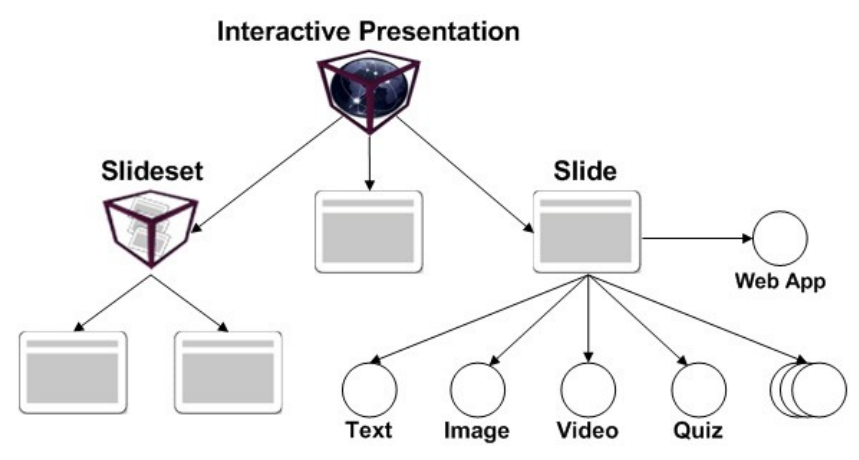

Fig. 1. Learning Object Model of ViSH Editor 
- The second level covers only one LO called "slide" that consists of a collection of level 1 LOs.

- The third level includes any LO that is built as a composition of level 2 LOs (i.e. "slides"). These LOs are identified under the name of "slidesets". Flashcards, Virtual Tours and Enriched Videos are examples of slidesets.

- Finally, the fourth level corresponds to the Interactive Presentation LO. An Interactive Presentation can contain level 3 LOs or directly a slide without using a slideset as a wrapper. Nevertheless, it cannot contain other Interactive Presentation (although it can contain their elements). These are the main differences with respect to the LOM specification.

The whole architecture of the system is composed by two main components: the viewer and the editor. The viewer is a fully client-side web application called ViSH Viewer [47] that allows users to view and interact with the created LOs. It is based on HTML5 [48], the new standard for the web. For this reason, any device with a HTML5 compatible web browser can run the tool and view the LOs without any installation being needed. The editor (i.e. ViSH Editor), is the web-based authoring tool where the authors create and edit the LOs. It is composed by two components: the client and the server backend. The client is a HTML5 web application that provides the user interface and most of the features of the editor. The server backend provides functionalities such as file uploading, file conversion (e.g. video, PDF), exporting and storing. An API has been defined to communicate the ViSH Editor client and the server. So, the tool is not tied to any specific backend technology, in fact, currently there are two different implementations of the server backend, one in Ruby on Rails and another one in Node.js. Moreover, ViSH Editor has an integration API to facilitate its integration and/or connection with LORs, allowing its use in different environments.

ViSH Editor is based on the WYSIWYG (What You See Is What You Get) paradigm and provides a usable and user friendly interface. It is internationalized and supports several languages such as English, Spanish, French and German. To create a LO, authors can insert many types of resources (e.g. images, videos, documents, websites, SCORM packages, etc.) via its URL, uploading them or by searching on different educational repositories and content providers such as Flickr, YouTube, SoundCloud or the LRE (Learning Resource Exchange) [49]. Besides adding resources, authors can type text and create several types of quizzes. ViSH Editor also facilitates authors to fill the metadata of the LOs. All LOs created by ViSH Editor and their metadata are saved in JSON format. However, the LOs can be exported to SCORM and their metadata can be retrieved in IEEE LOM facilitating their integration in Learning Management Systems. More details about ViSH Editor can be found in previous publications [50].

To this day, there is a public instance of ViSH Editor accessible through an e-Learning platform called Virtual Science Hub (ViSH, http://vishub.org) [15]. ViSH is fundamentally formed by a social network that aims to foster communication among teachers and scientists, a LOR that provides innovative educational resources, a videoconference service to perform virtual visits to scientific centers, and the ViSH Editor authoring tool that allows ViSH users to create and publish LOs. Both ViSH and ViSH Editor have been developed as part of the GLOBAL excursion European project.

\section{LEARNING OBJECTS}

The current version of ViSH Editor allows to create four different LOs: Flashcards, Virtual Tours, Enriched Videos and Interactive Presentations. The first three are slidesets since they belong to the third aggregation level of the LO model. On the other hand, Interactive Presentations belongs to the fourth level, and that implies that they can incorporate slidesets. ViSH Editor has been designed to be easy to extend with modules to create new slidesets. The inclusion of a new slideset, taken into account the LO model, not only increases the variety of educational resources that can be built, but also allows to enrich existing Interactive Presentations. All these LOs are provided as HTML5 applications and can be accessed from mobile devices. Moreover, they can be exported to SCORM (including their metadata in IEEE LOM format) and thus integrated in Learning Management Systems such as Moodle or Blackboard. The following sections describe each of these LOs, including an example of each. All the provided examples can be found in the ViSH repository.

\section{A. Flashcards}

Flashcards are resources presented as an image background with 'hot zones' identified by animated arrows on which the student can click to see additional contents that the teacher has previously linked. The additional contents are displayed in the form of slides. Thus, Flashcards link areas or Cartesian coordinates with slides. Authors can create the slides combining elements of the first aggregation level such as text, pictures, videos, quizzes, websites or web applications. Flashcards are very useful to mark different parts of infographics and provide extra information related with each of them. They can be used to explain the different parts of a microscope or the elements of an electronic circuit. An example of a Flashcard about the earth layers is displayed in Fig. 2. In this example, when students click on an arrow, a slide is displayed showing information about the pointed out earth layer. In the case of the crust, the slide contains a detailed explanation together with a video (Fig. 3).

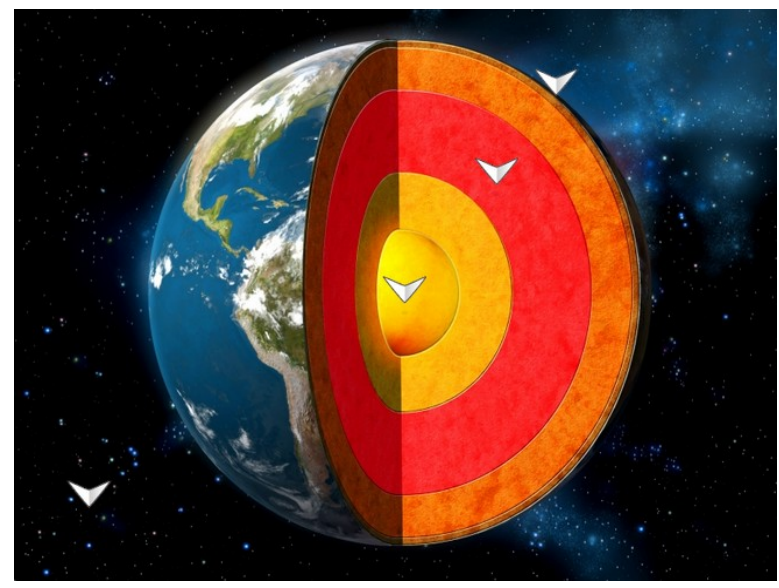

Fig. 2. Earth Layers Flashcard 
Crust

$\boldsymbol{\otimes}$

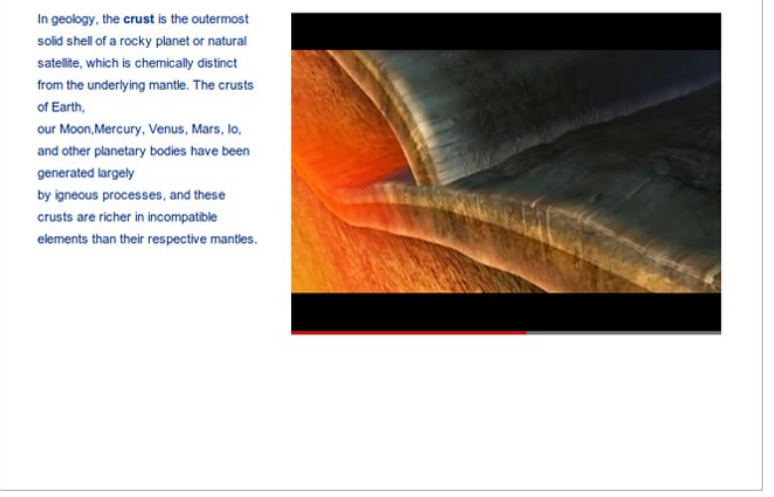

Fig. 3. Earth Layers Flashcard: The crust

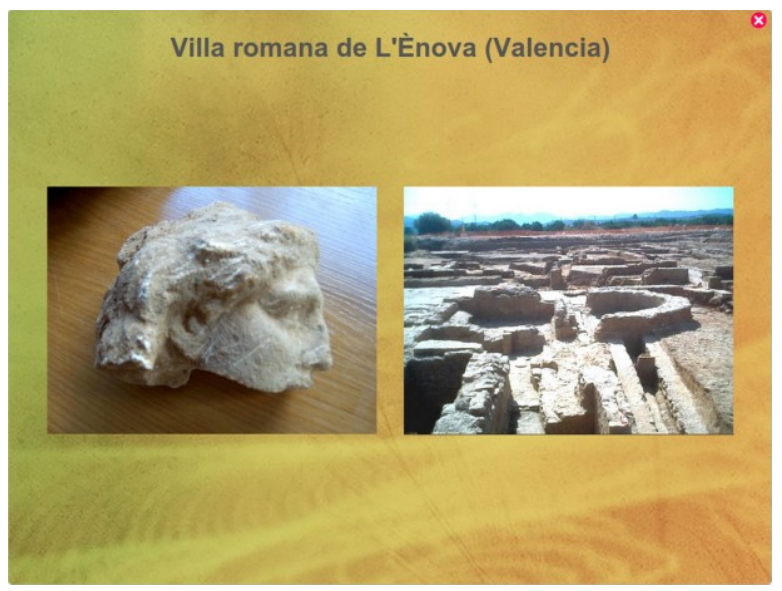

Fig. 5. Roman Villas Virtual Tour: L’Énova (Valencia)

\section{Enriched Videos}

Enriched Videos are videos augmented with other resources (e.g. quizzes, images, websites, etc.), which can be selected and customized by the author, and that can be displayed automatically when the video reaches a specific point of time or when the student requests one of them explicitly by selecting it in the progress bar or in a side menu. Optionally, Enriched Videos can also have chapters. The resources are represented through balls in the video progress bar while the chapters are represented with lines. Both resources and chapters may have an associated entry in the side menu. When students are watching an Enriched Video and the video reaches a point of time with a linked resource, the video will stopped and a new slide will appear showing the associated contents. When students close the slide or, in the case that the contents include some activity like a quiz, when they finish it, the video will resume automatically. Students can also select a specific content or jump to a specific point of time by clicking in the different entries of the side menu. Summarizing, Enriched Videos allow to link timestamps with slides. Although these LOs are generally built upon videos, they can also be built with audio files, allowing for instance, the creation of enriched audio podcasts. The only difference is that, in this case, the timestamps belong to an audio resource instead of a video. Authors can use both HTML5 and YouTube videos to create Enriched Videos. Another possibility that authors have is to upload their own videos (or audios) to the server backend to be converted to HTML5 compliant formats. The use of HTML5 media elements makes possible to ensure the accessibility from mobile devices. Enriched Videos have been designed with MOOCs (Massive Open Online Courses) [51] in mind. In this approach, they can be very useful since they enable to enrich video lectures with quizzes and to automatically track students' progress. Fig. 6 shows an Enriched Video used in a HTML5 MOOC offered by the Universidad Politécnica de Madrid. In this example, several quizzes are triggered on different moments of the video to check if the student has correctly understood the concepts explained in the video lecture.

Fig. 4. Roman Villas Virtual Tour 


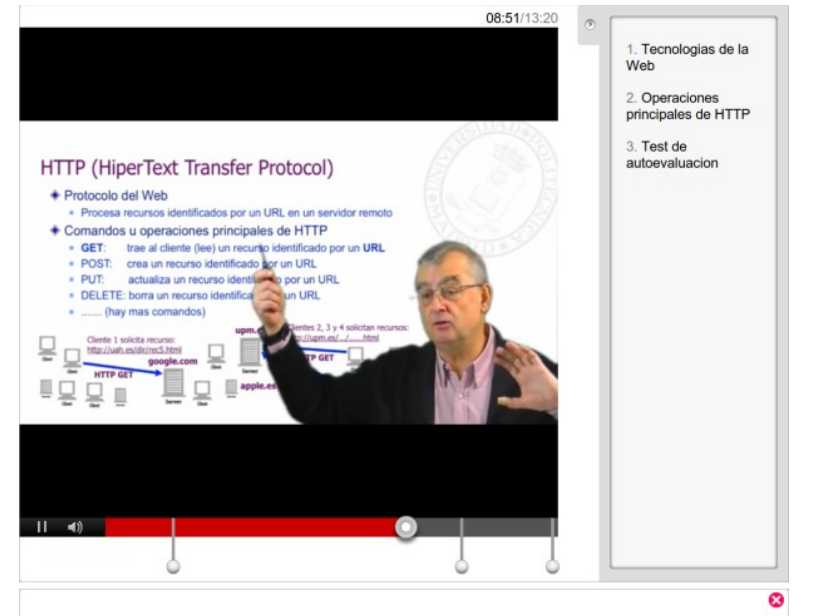

Indica cuáles de las siguientes afirmaciones son verdaderas o falsas. ฯ

\begin{abstract}
GET es un comando para traer páginas Web a un cliente para su visualización.

POST es un comando para editar recursos en un servidor.

PUT es un comando para crear recursos en un servidor.

DELETE es un comando para borrar recursos en un servidor.
\end{abstract}

Fig. 6. Enriched Video used in a HTML5 MOOC

\section{Interactive Presentations}

Interactive Presentations are series of slides displayed in a certain sequence, where each of these slides can be created by the author using different types of resources (text, multimedia, websites, SCORM packages, flash objects, quizzes, PDF files, etc.) including the Flashcards, Virtual Tours and Enriched Videos presented above. In other words, any element of the ViSH Editor Learning Object Model (Fig. 1) can be included. Thereby, a slide of an Interactive Presentation may display a slideset (third aggregation level) or a "slide" LO (second aggregation level). In general, students view the slides in sequential order and navigate through them using arrows, but authors can define links to connect one slide to another creating this way different learning paths. So, Interactive Presentations link slidesets and slides together to build static or adaptive LO sequences. Besides creating slides from scratch, authors can import PDF slideshows, SCORM packages, quizzes (e.g. from files in Moodle XML format) and also LOs of other Interactive Presentations fostering their reuse. This is the start LO of the ViSH repository, where it has been renamed as Virtual Excursion. There are hundreds of these LOs public available on the ViSH, covering a lot of topics such as computer science, software engineering, technology, maths, physics, biology, natural sciences, foreign languages or chemistry. Interactive Presentations have been found useful to enrich existing educational resources producing this way LOs with higher quality. For instance, some teachers have created high quality
LOs by using interactive simulations (e.g. PhET creative commons simulations [52]) together with a series of activities and quizzes related to them. Other remarkable components that have been used to create LOs are the e-Infrastructure resources. These resources are one of the exclusive characteristics of ViSH and are offered by e-Infrastructure providers, which are partners of the GLOBAL excursion project. An example of an e-Infrastructure resource can be a webcam of a natural park, a microscope, or a pulley of a remote physics laboratory. The screenshots of Fig. 7 correspond to an Interactive Presentation that uses one of these resources, a pendulum provided by the remote physics laboratory of the Institute for Biocomputation and Physics of Complex Systems, located in Zaragoza, Spain. The presentation begins with an explanation of the main physics concepts related to the simple pendulum, including all the information and mathematical formulas required to perform the future activities. Some solved exercises are also included. Then, students have to answer several quizzes and do some exercises related to the contents previously explained. After that, a practice is described, in which the students have to perform an experiment using the real pendulum and compare their theoretical calculations with the measurements obtained from the real system. Besides the real pendulum, the presentation also includes a pendulum simulation provided by $\mathrm{PhET}$. This is very useful if there are not enough real pendulums for all students or to compare the results of the same experiments using a real and a virtual pendulum. Lastly, Fig. 8 shows a screenshot of ViSH Editor when editing this Interactive Presentation.

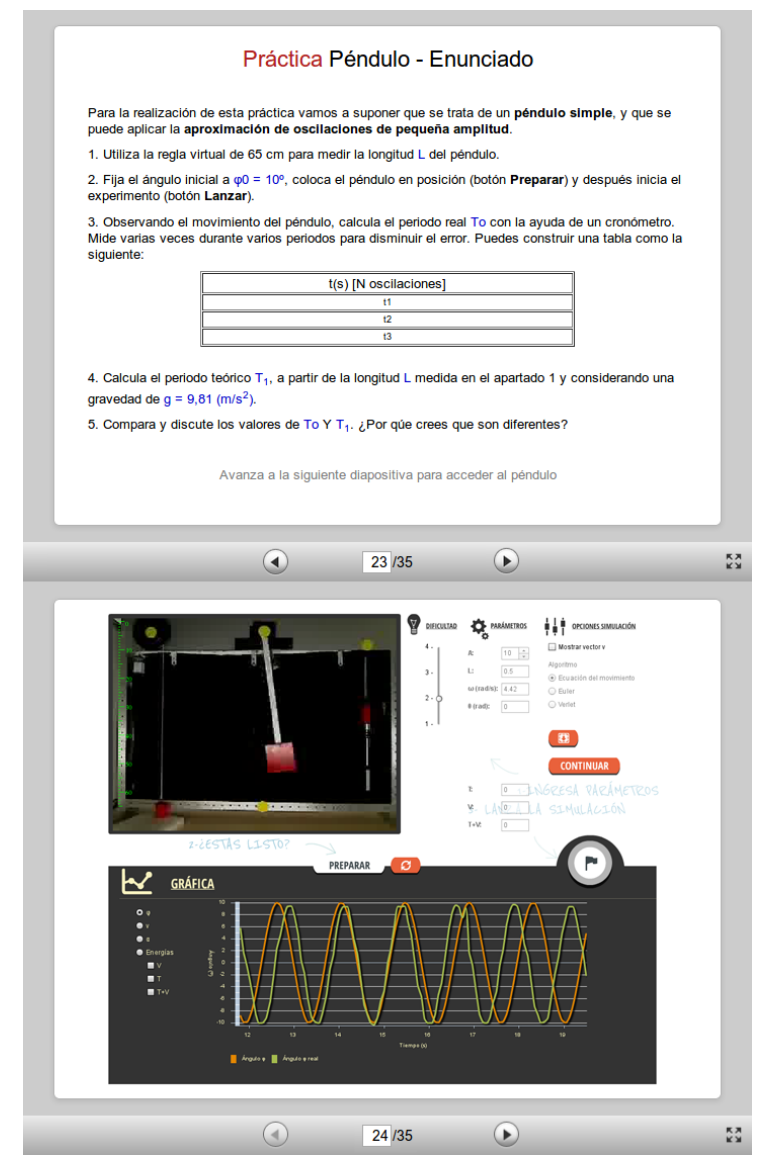

Fig. 7. Interactive Presentation: Introduction to the Simple Pendulum 


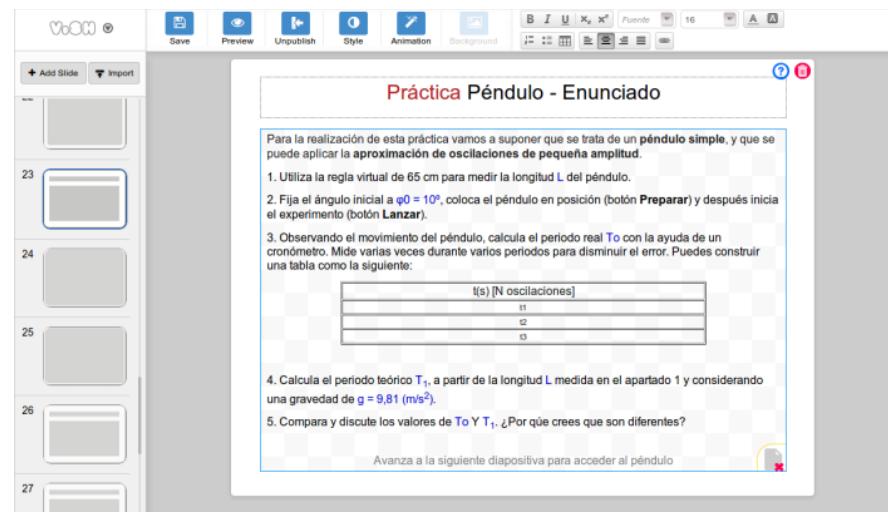

Fig. 8. User Interface of ViSH Editor

\section{EVAlUATION}

The success of this approach for creating LOs will depend on two main factors: the usability of the authoring tool and the quality as educational resources of the created LOs.

\section{A. Authoring Tool Evaluation}

To evaluate the ViSH Editor authoring tool, a 13 questions survey was conducted to collect authors' feedback on overall opinion, usability and proposed changes among others. ViSH Editor has been used by different types of author: primary and secondary school teachers, lecturers, researchers, student teachers and even high school students and undergraduates. With the aim of evaluating the tool for teachers, students and undergraduates were excluded from the study. An email with the online survey link was sent on December 2013 to all ViSH teachers (as well as student teachers) that had created and published at least one $\mathrm{LO}$ in the ViSH platform during the last three months. A total of 67 surveys were collected. The sample consisted of 67 teachers, 34 males $(50.7 \%)$ and 33 females $(49.3 \%), 19$ to 65 years of age $(\mathrm{M}=35.9, \mathrm{SD}=13.1)$.

Survey results show that $29.9 \%$ of the authors who filled the survey had an "Excellent" overall opinion of ViSH Editor, 61.2\% "Good", 7.5\% "Neutral", 1.5\% "Bad" and that nobody had an "Awful" opinion. The perceived usability was obtained from the question "How would you describe the experience of learning to use ViSH Editor?" in which authors answered on a scale from 1 to 5 with 1 being "very difficult" and 5 being "very easy". This question recorded a mean of 4.2 and a standard deviation of $0.8(\mathrm{M}=4.2, \mathrm{SD}=0.8)$, showing that authors found ViSH Editor easy to use.

Respondents were also asked about their overall experience when creating the different LOs. They answered this question on a scale from 1 to 5 with 1 being "Awful" and 5 being "Excellent". Table I summarizes the results. All creation tasks obtained an overall experience rating higher than 4 out of 5 with low standard deviation. No significant differences were found on the authors overall experience when performing the different creation tasks.
TABLE I. AUTHOR'S OVERALL EXPERIENCE RATING

\begin{tabular}{|c|c|c|}
\hline Task & M & SD \\
\hline Creation of Flashcards & 4.1 & 1.1 \\
\hline Creation of Virtual Tours & 4.2 & 0.9 \\
\hline Creation of Enriched Videos & 4.2 & 0.7 \\
\hline Creation of Interactive Presentations & 4.2 & 0.4 \\
\hline
\end{tabular}

\section{B. Learning Objects Evaluation}

To evaluate the quality of the LOs created with ViSH Editor, we used a formal LO evaluation model called LORI (Learning Object Review Instrument) [16]. LORI is one of the better known LO evaluation models and has been tested in a few studies ([53], [54]), which have shown that it can be used to reliably assess some aspects of LOs. We used the version 1.5 of LORI, which defines 9 items for LO evaluation related to the following criteria: Content Quality (item 1), Learning Goal Alignment (2), Feedback and Adaptation (3), Motivation (4), Presentation Design (5), Interaction Usability (6), Accessibility (7), Reusability (8), and Standards Compliance (9). Reviewers have to rate each item using a 5-point scale and also they can provide comments with their reviews. A total of 209 Interactive Presentations were evaluated by 15 reviewers using an online version of LORI, generating a total amount of 740 evaluations. The group of reviewers was composed by 4 e-Learning professionals, 9 educators and 2 designers. Each LO was evaluated by at least 3 reviewers. A "LORI Weighted Arithmetic Mean metric" was used as quality metric. This metric calculates the score of a LO as the weighted arithmetic mean of all LORI items scores, giving different importance to each criteria. The set of weights for the different criteria were obtained through a survey among the reviewers obtaining the following result: $\mathrm{W}_{1}=0.1724, \mathrm{~W}_{2}=0.1207, \mathrm{~W}_{3}=0.1138$, $\mathrm{W}_{4}=0.1414, \mathrm{~W}_{5}=0.1379, \mathrm{~W}_{6}=0.1034, \mathrm{~W}_{7}=0.0655, \mathrm{~W}_{8}=0.0759$, $\mathrm{W}_{9}=0.069$, where $\mathrm{W}_{\mathrm{i}}$ corresponds to the weight of the LORI item $\mathrm{i}$. The equation that calculates the LO score takes an input score vector $\left\{i_{1}, \ldots, i_{9}\right\}$, being $i_{i}$ the score of the LORI item $i$ in a scale from 1 to 5 , and yields a single real value in a scale from 0 to 10 according to the following expression:

$$
\begin{gathered}
s\left(\left\{i_{1}, \ldots, i_{9}\right\}\right)=\frac{5}{2} \times \sum_{i=1}^{9} W_{i} \times\left(i_{i}-1\right), s(\{i\}) \in[0,10] \\
\text { where } \sum_{i=1}^{9} W_{i}=1 \text { and } W_{i} \geq 0 \forall i \in\{1, \ldots, 9\}
\end{gathered}
$$

When using this quality metric, a score value of 5 may be used as threshold value to reliably distinguish between low and high quality LOs [55]. That implies that LOs scored higher than 5 can be considered of enough quality to be used for education.

Based on this quality metric, four groups of Interactive Presentations were compared (see Table II). The first group was formed by presentations that include Flashcards $(\mathrm{M}=7.0$, $\mathrm{SD}=1.3)$, the second one by presentations that include Virtual Tours $(\mathrm{M}=6.7, \mathrm{SD}=0.7)$, the third one by presentations that include Enriched Videos $(\mathrm{M}=7.6, \mathrm{SD}=0.8)$, and the last group includes all the evaluated LOs $(\mathrm{M}=6.0, \mathrm{SD}=1.6)$. 
TABLE II. AVERAGE QUALITY SCORE OF VISH EDITOR LOS

\begin{tabular}{|c|c|c|}
\hline \multirow{2}{*}{ Learning Objects } & \multicolumn{2}{|c|}{ Quality Score } \\
\cline { 2 - 3 } & $\boldsymbol{M}$ & $\boldsymbol{S D}$ \\
\hline Presentations with Flashcards & 7.0 & 1.3 \\
\hline Presentations with Virtual Tours & 6.7 & 0.7 \\
\hline Presentations with Enriched Videos & 7.6 & 0.8 \\
\hline All Interactive Presentations & 6.0 & 1.6 \\
\hline
\end{tabular}

These results show that presentations with slidesets tend to have higher quality. The Flashcard group recorded an average quality score $16.3 \%$ higher than the general group. The same occurs with the Virtual Tour group (11.5\%) and the Enriched Video group (26.5\%). The average quality recorded by the general group was 6.0. Given that this score exceeds the quality threshold of 5, we can affirm that on average the LOs generated through ViSH Editor have good quality. However, a standard deviation value of 1.6 denotes that exist a significant quality difference among the evaluated LOs. The analysis of the quality score distribution among the LOs confirmed this fact. In this study the maximum scored achieved by a LO was 9.34 and the minimum was 0.26 . These empirical evidences reinforce the idea that authoring tools can facilitate the creation of high quality LOs, but they cannot guarantee it.

Finally, a general ViSH survey was conducted among all ViSH users in January 2014. In this case, the sample consisted of 97 users and no demographic data were provided. In one of the survey questions users were asked to rate the usefulness as educational resources of the different LOs that can be created with ViSH Editor using a scale from 1 to 5 with 1 being "Not Useful" and 5 being "Very Useful". Table III shows the results of this question. All LOs were rated higher than 4 out of 5 with low standard deviations.

TABLE III. PERCEIVED USEFULNESS AS EDUCATIONAL RESOURCES OF VISH EDITOR LOS

\begin{tabular}{|c|c|c|}
\hline Learning Objects & M & SD \\
\hline Flashcards & 4.3 & 0.8 \\
\hline Virtual Tours & 4.4 & 0.7 \\
\hline Enriched Videos & 4.5 & 0.7 \\
\hline Interactive Presentations & 4.6 & 0.6 \\
\hline
\end{tabular}

\section{CONCLUSIONS AND FUTURE WORK}

This paper presents an open source online e-Learning authoring tool for creating interactive multi-device LOs. Four distinct LOs have been described: Flashcards, Virtual Tours, Enriched Videos and Interactive Presentations, including their possible uses in educational settings and an example of each. All these LOs are web applications that can be accessed via mobile devices. Besides, they can be exported to SCORM including their metadata in IEEE LOM format. This approach for creating LOs was validated through two evaluations: a survey among authors and a formal quality evaluation of a set of 209 LOs created with the tool. The results show that ViSH Editor facilitates educators the creation of high quality LOs.
The work exposed in this paper show that educators can create high quality courseware in a cost-effective way if they have the suitable authoring systems. To be successful, these systems need to be easy to use and able to generate quality outputs with little effort. Nevertheless, there is no authoring tool that can guarantee that the educational resources it creates have a high quality, although they can help to make this happen.

Another finding of this study is that educational resources that include interactive LOs like Flashcards, Virtual Tours or Enriched Videos, tend to have higher quality. Thus, more work should be done to facilitate the creation and reuse of new LOs of that type. For this reason, we plan to extend ViSH Editor with new modules to allow the creation of new LOs such as 3D Flashcards or augmented reality resources. Further research is also recommended into LO quality indicators as well as instructional strategies to use LOs.

\section{ACKNOWLEDGMENT}

We wish to acknowledge our gratitude and appreciation to all the GLOBAL excursion project partners, and each one of the project team members, for their contribution to this paper.

\section{REFERENCES}

[1] D. A. Wiley, "Learning Object Design and Sequencing Theory," Brigham Young University, 2000.

[2] E. Duval, K. U. Leuven, and W. Hodgins, "A LOM Research Agenda," in Proceedings of the 12th International World Wide Web Conference (2003), 2003, pp. 1-9.

[3] R. McCormick and N. Li, "An evaluation of European learning objects in use," Learning, Media and Technology, vol. 31, no. 3, 2006.

[4] S. Nurmia and T. Jaakkolaa, "Effectiveness of learning objects in various instructional settings," Learning, Media and Technology, vol. 31, no. 3, pp. 233-247, 2006.

[5] Y. Akpinar and H. Simsek, "Should K-12 Teachers Develop Learning Objects? Evidence from the Field with K-12 Students," International Journal of Instructional Technology and Distance Learning, 2007.

[6] R. Kay, "Examining the Effectiveness of Web-Based Learning Tools in Middle and Secondary School Science Classrooms," Interdisciplinary Journal of E-Learning and Learning Objects, vol. 7, 2011.

[7] R. Kay, "Exploring the use of web-based learning tools in secondary school classrooms," Interactive Learning Environments, Jan. 2012.

[8] J. C. Nesbit, T. L. Leacock, C. Xin, and G. Richards, "Learning Object Evaluation and Convergent Participation: Tools for Professional Development in E-Learning," in Proceedings of the IASTED International Conference on Computers and Advanced Technology in Education, 2004.

[9] T. Leacock, G. Richards, and J. C. Nesbit, "Teachers need simple, effective tools to evaluate learning objects: Enter eLera.net," in Proceedings of the Seventh IASTED International Conference on Computers and Advanced Technology in Education, 2004.

[10] X. Ochoa and E. Duval, "Relevance Ranking Metrics for Learning Objects," IEEE Transactions on Learning Technologies, vol. 1, no. 1, pp. 34-48, 2008.

[11] N. Y. Yen, T. K. Shih, L. R. Chao, and Q. Jin, "Ranking Metrics and Search Guidance for Learning Object Repository," IEEE Transactions on Learning Technologies, vol. 3, no. 3, pp. 250-264, 2010.

[12] J. Sanz-Rodriguez, J. M. Dodero, and S. Sanchez-Alonso, "Ranking Learning Objects through Integration of Different Quality Indicators," IEEE Transactions on Learning Technologies, vol. 3, no. 4, pp. 358-363, 2010.

[13] D. Gallego, E. Barra, S. Aguirre, and G. Huecas, "A Model for Generating Proactive Context-Aware Recommendations in e-Learning 
Systems," in Proceedings of the 2012 Frontiers in Education Conference (FIE 2012), 2012.

[14] D. Gallego, E. Barra, A. Gordillo, and G. Huecas, "Enhanced Recommendations for e-Learning Authoring Tools based on a Proactive Context-aware Recommender," in Proceedings of the 2013 Frontiers in Education Conference (FIE 2013), 2013.

[15] E. Barra, A. Gordillo, and J. Quemada, "Virtual Science Hub: An Open Source Platform To Enrich Science Teaching," in Proceedings of the International Conference on Educational Sciences and Technology (ICEST 2014), 2014.

[16] T. L. Leacock and J. C. Nesbit, "A Framework for Evaluating the Quality of Multimedia Learning Resources," Educational Technology and Society, vol. 10, pp. 44-59, 2007.

[17] Webopedia: Online Computer Dictionary For Computer And Internet Terms And Definitions, [Online]. Available: http://www.webopedia.com/TERM/A/authoring_tool.html.

[18] J. Preclík, "Authoring Tools," in the 9th Annual Conference of Doctoral Students, 2000.

[19] J. Harris, "An Introduction to Authoring Tools," ASTD's Learning Circuits online magazine, 2002.

[20] T. Murray, B. Stephen, and S. Ainsworth, Authoring tools for advanced technology learning environments: Toward cost-effective adaptive, interactive and intelligent educational software. Springer, 2003.

[21] "Advanced Distributed Learning (ADL), SCORM 2004 4th Edition," 2004. [Online]. Available: http://www.adlnet.gov/capabilities/scorm/scorm-2004-4th.

[22] J. Poltrack, N. Hruska, A. Johnson, and J. Haag, "The Next Generation of SCORM: Innovation for the Global Force," in Proceedings of the Interservice/Industry Training, Simulation, and Education Conference (I/ITSEC) 2012, 2012.

[23] K. M. Al-shawkani, "E-Learning Authoring Tools: The Present and Future Vision," in Proceedings of 3rd Annual Forum on e-Learning Excellence in the Middle East, 2010.

[24] J. Preclík, "What authoring tool is the best?," in the 11th Annual Conference of Doctoral Students, 2002.

[25] T. H. Kaskalis, T. D. Tzidamis, and K. Margaritis, "Multimedia Authoring Tools: The Quest for an Educational Package," Journal of Educational Technology \& Society, vol. 10, no. 3, 2007.

[26] M. Haghshenas, M. Khademi, and H. Kabir, "E-learning and Authoring Tools: At a Glance," International Journal of Research \& Reviews in Applied Sciences, vol. 10, no. 2, pp. 259-263, 2012.

[27] “Articulate Quizmaker.” [Online]. Available: http://articulate.com/products/quizmaker.php.

[28] "Microsoft PowerPoint." [Online]. Available: http://office.microsoft.com/powerpoint.

[29] S. Ainsworth and P. Fleming, "Evaluating authoring tools for teachers as instructional designers," Computers in Human Behavior, vol. 22, no. 1, pp. 131-148, Jan. 2006.

[30] E. Barra, A. Gordillo, D. Gallego, and J. Quemada, "Integration of SCORM packages into web games," in Proceedings of the 2013 Frontiers in Education Conference (FIE 2013), 2013.

[31] "Easygenerator." [Online]. Available: http://www.easygenerator.com.

[32] C. D. Milligan, P. Beauvoir, and P. Sharples, "The Reload Learning Design Tools," Journal of Interactive Media in Education, no. 1, 2005.

[33] J. Watson, A. Dickens, and G. Gilchrist, "The LOC Tool: Creating a Learning Object Authoring Tool for Teachers," in Proceedings of Ed-Media 2008: World Conference on Educational Multimedia, Hypermedia \& Telecommunications., 2008.

[34] T. Boyle, "Generative learning objects (GLOs): design as the basis for reuse and repurposing," in First International Conference of e-Learning and Distance Education, 2009.
[35] A. Koohang, K. Floyd, and C. Stewart, "Design of an Open Source Learning Objects Authoring Tool - The LO Creator," Interdisciplinary Journal of E-Learning and Learning Objects, vol. 7, 2011.

[36] "Educaplay." [Online]. Available: http://www.educaplay.com.

[37] R. Mcgreal, "Learning Objects: A Practical Definition," International Journal of Instructional Technology and Distance Learning, vol. 1, no. 9, pp. 21-32, 2004.

[38] D. A. Wiley, "Connecting learning objects to instructional design theory: A definition, a metaphor, and a taxonomy," in The instructional use of learning objects, 2000.

[39] K. Chitwood (Wisconsin Online Resource Center), "Learning Objects: Making a Difference in Teaching and Learning," in 20th Annual Conference on Distance Teaching and Learning, 2005, pp. 1-5.

[40] J. Mcdonald, "Learning object: A new definition , a case study and an argument for change," in Proceedings of the 23rd annual ascilite conference: Who's learning? Whose technology?, 2006, pp. 535-544.

[41] S. Fadzilah, N. Yusof, and S. Zaiton, "Creating Granular Learning Object Towards Reusability of Learning Object In E-learning Context," in Proceedings of the 2011 International Conference on Electrical Engineering and Informatics, 2011.

[42] A. Littlejohn, I. Falconer, and L. Mcgill, "Characterising effective eLearning resources," Computers \& Education, vol. 50, no. 3, pp. 757-771, Apr. 2008.

[43] S. Schluep, "Modularization and structured markup for Web-based Learning Content in an Academic Environment," 2005.

[44] "Dublin Core Metadata." [Online]. Available: http://www.dublincore.org/.

[45] IEEE LTSC, "Draft Standard for Learning Object Metadata (IEEE LOM)," 2002.

[46] “CanCore Learning Resource Metadata Initiative." [Online]. Available: http://cancore.athabascau.ca/en.

[47] A. Gordillo, E. Barra, and J. Quemada, "Enhancing K-12 science education through a multi-device web tool to facilitate content integration and e-Infrastructure access," in Proceedings of the 7th International Technology, Education and Development Conference (INTED 2013), 2013.

[48] W3C, "HTML5 specification." [Online]. Available: http://www.w3.org/html/wg/drafts/html/master/Overview.html.

[49] "Learning Resource Exchange (LRE)." [Online]. Available: http://lre.eun.org.

[50] A. Gordillo, E. Barra, D. Gallego, and J. Quemada, "An online e-Learning authoring tool to create interactive multi-device learning objects using e-Infrastructure resources," in Proceedings of the 2013 Frontiers in Education Conference (FIE 2013), 2013, pp. 1914-1920.

[51] A. Mcauley, B. Stewart, G. Siemens, and D. Cormier, "The MOOC model for digital practice." 2010.

[52] "PhET Interactive Simulations." [Online]. Available: https://phet.colorado.edu.

[53] J. Vargo, J. C. Nesbit, K. Belfer, and A. Archambault, "Learning object evaluation: computer-mediated collaboration and inter-rater reliability," International Journal of Computers and Applications, vol. 25, no. 3, 2003.

[54] F. Krauss and M. Ally, "A Study of the Design and Evaluation of a Learning Object and Implications for Content Development," Interdisciplinary Journal of E-Learning and Learning Objects, vol. 1, pp. 1-22, 2005.

[55] A. Gordillo, E. Barra, and J. Quemada, "Towards a Learning Object pedagogical quality metric based on the Learning Object Review Instrument," in Proceedings of the 2014 Frontiers in Education Conference (FIE 2014), 2014. 